\title{
The Effects of Supraphysiologic Doses of Vitamin D3 in Conjunction With Teriparatide on Bone Mineral Density in Two Postmenopausal Females With Severe Osteoporosis
}

\author{
Pooja Raghavan ${ }^{a}$, Elena Christofides ${ }^{\mathrm{b}, \mathrm{c}}$
}

\begin{abstract}
Teriparatide was approved by the FDA in 2002 for the treatment of osteoporosis in postmenopausal women who are at high risk for fractures. Previous studies involving the use of teriparatide in conjunction with vitamin D and calcium supplementation have suggested that sufficient vitamin D levels may not be a requirement to achieve an effective response to teriparatide. We present a case of two postmenopausal females with severe osteoporosis who were treated with teriparatide along with calcium and supraphysiologic doses of vitamin D, as illustrated by an increase from their baseline serum 25-hydroxyvitamin D concentrations. Both patients experienced an increase in bone mineral density that was significantly higher than what has been seen in prior studies of teriparatide administration in conjunction with physiologic doses of vitamin D. These findings suggest that administering supraphysiologic doses of vitamin D may in fact potentiate the effects of teriparatide in postmenopausal females with osteoporosis, resulting in larger increases in bone mineral density than would otherwise be expected.
\end{abstract}

Keywords: Osteoporosis; Teriparatide; Fracture

\section{Introduction}

Osteoporosis currently affects 10 million people in the United States, with 18 million more still at risk [1]. Due to the increasing number of patients being diagnosed annually with

\footnotetext{
Manuscript accepted for publication June 20, 2012

${ }^{a}$ Department of Medicine, Mount Carmel Health, Columbus, Ohio, United States

${ }^{\mathrm{b}}$ Department of Medicine, Division of Endocrinology, Mount Carmel Health, Columbus, Ohio, United States

${ }^{\mathrm{c}}$ Corresponding author: Elena A Christofides, 72 West 3rd Ave,

Columbus, OH 43201, United States.

Email: christofides@endocrinology-associates.com
}

doi: $10.4021 /$ jem $104 \mathrm{w}$ this disabling disease, new therapies are continuing to be studied with the aim of increasing bone mineral density and reducing the risk of fractures.

Antiresorptive agents have been widely studied in the treatment of postmenopausal osteoporosis; including bisphosphonates, estrogen, raloxifene, and calcitonin. Teriparatide was approved by the FDA in 2002 for the treatment of osteoporosis in postmenopausal women at high risk for fractures [2]. Calcium and vitamin D are an essential component of the treatment of osteoporosis, and while alone they may not significantly affect bone mineral density, an adequate dose of both complements the anti-fracture efficacy of the antiresorptive and anabolic agents [3]. Previous trials involving teriparatide therapy supplemented with vitamin D and calcium involve doses of vitamin D no higher than 1200 IU/day or with the aim of achieving a serum 25-hydroxyvita$\min \mathrm{D}$ level of $50 \mathrm{nmol} / \mathrm{L}$ [4]. No studies to date have shown the effects of supraphysiologic doses of vitamin D supplementation in conjunction with teriparatide therapy on bone mineral density and fracture prevention.

We present 2 cases of postmenopausal females with severe osteoporosis who were treated with supraphysiologic doses of vitamin D throughout their treatment period on teriparatide, and report results of 25-hydroxyvitamin D levels and bone mineral density in response to such therapy.

\section{Case Presentation}

\section{Case 1}

A 68-year old postmenopausal Caucasian female with a history of premature menopause at age 38 was referred for management of osteoporosis. The patient had never been placed on hormone replacement therapy and had no history of fractures. She had a recent dual-energy x-ray absorptiometry (DEXA) showing a bone mineral density (BMD) of $0.393 \mathrm{~g} /$ $\mathrm{cm}^{2}$ and a T-score of -5.9 at the lumbar spine. Prior to evaluation at our clinic, the patient had been started on alendronate and $2800 \mathrm{IU} /$ day of vitamin $\mathrm{D}$, which she continued for one month before being seen by our team. The patient's height was measured at 58 inches, though she stated a previous 
height of 66 inches approximately 10 years prior. Given the severity of osteoporosis, the patient was started on teriparatide, calcium citrate $2000 \mathrm{mg} /$ day, and vitamin D3 50,000 IU weekly. Her previous therapy with alendronate and vitamin $\mathrm{D}$ was discontinued.

The patient continued this medication regimen without any changes or additions for 24 months. Serum 25-hydroxyvitamin $\mathrm{D}$, calcium, phosphorus, intact $\mathrm{PTH}$, and urine cross-linked $\mathrm{N}$-telopeptide levels were checked at baseline and at 12-month intervals while on teriparatide therapy (Table 1). TSH and albumin were also checked, which were in normal range at 1.6 (reference range: $0.5-4.8$ ) and 3.5 (reference range: 3.5 - 4.8), respectively. Renal function, glomerular filtration rate, and 24-hour urine calcium were measured at baseline and 12-month intervals while on therapy. These remained within normal limits throughout the 24 months of therapy. The 1, 25-dihydroxyvitamin D concentration was 56 (reference range: 25 - 66) at 12 months. 25-hydroxyvitamin $D$ levels exhibited a significant change from baseline. At the end of two years, a DEXA scan was repeated which showed a BMD of $0.582 \mathrm{~g} / \mathrm{cm}^{2}$ and a T-score of -4.2 at the lumbar spine (Table 2). This was a $48.2 \%$ change from baseline values. The patient sustained no fractures and no further loss of height during the treatment period on teriparatide.

\section{Case 2}

A 67-year old postmenopausal Caucasian female with a history of menopause at age 43 was referred for management of severe osteoporosis. Her history was notable for seven vertebral fractures and loss of height in all of the lumbar vertebral bodies since menopause. Initial DEXA two months prior revealed a BMD of $0.351 \mathrm{~g} / \mathrm{cm}^{2}$ and a T-score of -6.3 at the lumbar spine. The patient had never been placed on hormone replacement therapy, but had been started on alendronate and vitamin D 5,600 IU/day after results of DEXA became available. On physical examination, the patient appeared frail, with a height of 59 inches. She reported a height of 63 inches prior to menopause. Due to a new intertrochanteric fracture of the left hip while on alendronate therapy, her prior treatment was discontinued, and the patient was started on teriparatide, calcium citrate $2,000 \mathrm{mg}$ /day and vitamin D3 50,000 IU weekly.

The patient continued this medication regimen without any changes or additions for 24 months. Serum 25-hydroxyvitamin D, calcium, phosphorus, intact PTH, and urine cross-linked N-telopeptide levels were checked at baseline and at 12-month intervals while on teriparatide therapy (Table 1). TSH and albumin were also checked, which were in normal range at 0.74 (reference range: $0.5-4.8$ ) and 3.9 (reference range: 3.5 - 4.8), respectively. Renal function, glomerular filtration rate, and 24-hour urine calcium were measured at baseline and 12-month intervals while on therapy. These remained within normal limits throughout the 24

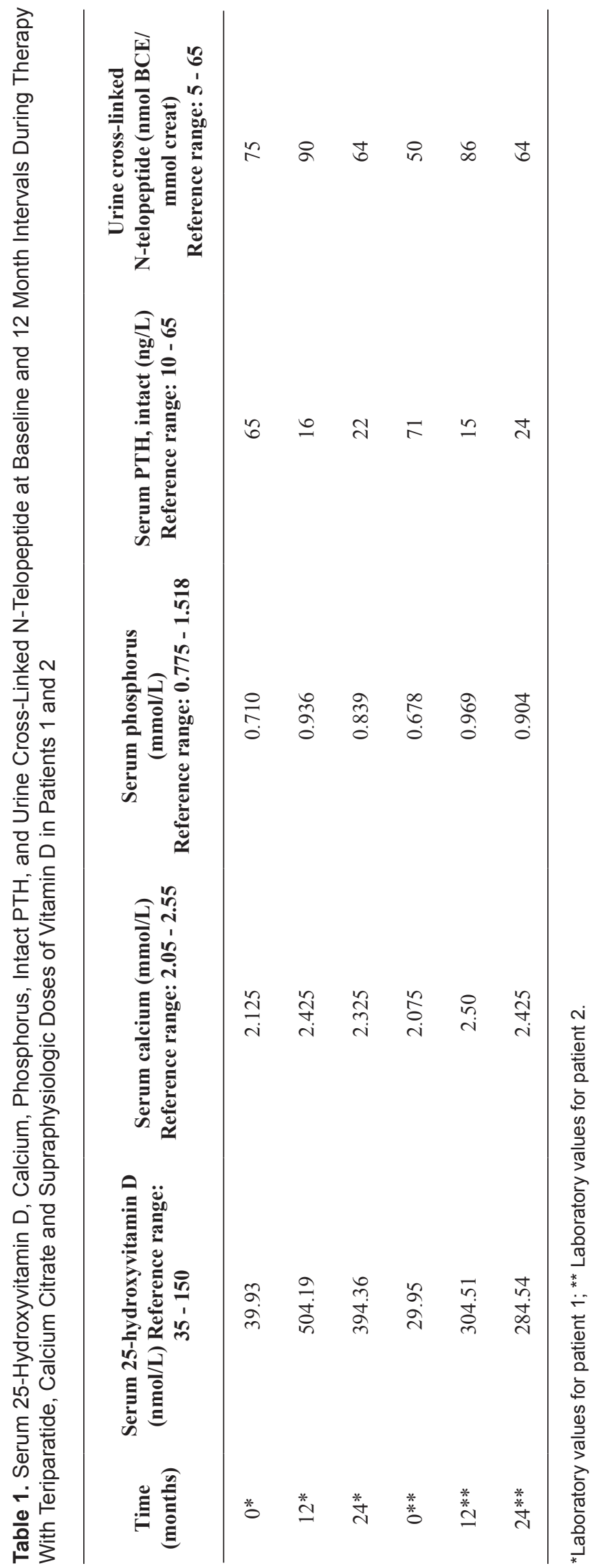


Table 2. Comparison of BMD and T-Scores Prior to and 24 Months After Treatment With Teriparatide, Calcium Citrate and Supraphysiologic Doses of Vitamin D in Patients 1 and 2, Percent Change and Absolute Increase in BMD is Also Noted

\begin{tabular}{lcc}
\hline & Patient 1 & Patient 2 \\
\hline BMD, L1-L4 $\left(\mathrm{g} / \mathrm{cm}^{2}\right)$, baseline & 0.393 & 0.351 \\
BMD, L1-L4 $\left(\mathrm{g} / \mathrm{cm}^{2}\right)$, at 24 months & 0.582 & 0.639 \\
T-score, L1-L4, baseline & -5.9 & -6.3 \\
T-score, L1-L4, at 24 months & -4.2 & -3.7 \\
\% change in BMD, L1-L4, at 24 months & $48.2 \%$ & $82.3 \%$ \\
Absolute increase in BMD at 24 months $\left(\mathrm{g} / \mathrm{cm}^{2}\right)$ & 0.189 & 0.288 \\
\hline
\end{tabular}

months of therapy. The 1, 25-dihydroxyvitamin D concentration was 49 (reference range: 25 - 66) at 12 months. 25-hydroxyvitamin D levels exhibited a significant change from baseline. At the end of two years, a DEXA scan was repeated which revealed a BMD of $0.639 \mathrm{~g} / \mathrm{cm}^{2}$ and a T-score of -3.7 at the lumbar spine (Table 2). This was an $82.3 \%$ change from baseline values. The patient sustained no new fractures during the treatment period on teriparatide.

The DEXA results in case 1 and 2 exhibit a 4 to 8 fold increase in bone mineral density at the lumbar spine respectively, in comparison to results from the largest double-blind, multicenter, placebo-controlled clinical trial involving teriparatide administration in conjunction with physiologic doses of vitamin D supplementation (Table 3) [2].

\section{Discussion}

Osteoporosis is a disease in which bone quality and strength become compromised, leading to an increased risk of fractures [1]. The risk of osteoporosis increases with age, and it is estimated that 1 in 3 patients who sustain an osteoporotic hip fracture are sent to a nursing home within a year [1]. Due to the disabling nature of the disease and the significant increases in morbidity and mortality associated with osteoporotic fractures, drug therapies continue to be studied with the aim of increasing bone mineral density and subsequently reducing the risk of fractures in patients with osteoporosis [5].

Teriparatide, a recombinant human parathyroid hormone analog 1 - 34, was approved by the FDA in 2002 for the treatment of osteoporosis in postmenopausal women who are at high risk for fractures [2]. Hodsman et al suggest that patients who are considered high risk for osteoporotic fractures include: patients with very low bone density (T-score $\leq-3.5$ ), with preexisting fractures, or with a suboptimal response to antiresorptive therapy [6]. Teriparatide is regarded as being the first and only anabolic agent available for the treatment of osteoporosis. Its mechanism of action differs from other agents currently used. Historically, chronic exposure to PTH has been observed to lead to osteoporosis, as in diseases such as hyperparathyroidism [7]. This is due to PTH induced stimulation of osteoclasts, leading to an increase in bone resorption [7]. Teriparatide, however, reaches peak serum concentrations in 30 minutes and returns to non-detectable serum levels within a few hours [7]. This intermittent burst of PTH given in the form of teriparatide, in contrast to chronic exposure to $\mathrm{PTH}$, has the paradoxical effect of stimulating new bone formation and increasing bone mass by favoring stimulation of osteoblasts more than osteoclasts [7].

Calcium and vitamin D are also essential components of the treatment of osteoporosis. Calcium is required to attain peak bone mass, while vitamin $\mathrm{D}$ is required to optimize calcium absorption [1]. Vitamin D is acquired through direct exposure to sunlight and by diet, and its ability to increase the 25-hydroxyvitamin D level is affected by many different factors; this makes it difficult to standardize requirements [8]. Recent studies have suggested that the current daily requirements for vitamin $\mathrm{D}$ are inadequate, and concentrations of 25-hydroxyvitamin D less than $80 \mathrm{nmol} / \mathrm{L}$ are considered deficient [9]. Circulating 25-hydroxyvitamin D levels less than $80 \mathrm{nmol} / \mathrm{L}$ have been shown to cause a reduction in the absorption of calcium, resulting in osteoporosis and an increased risk of fractures [10-11]. Recently, the Institute of Medicine recommended an increase in the daily requirement of vitamin D, based on an extensive review of studies on health outcomes [8]. The evidence supported a definite role for calcium and vitamin $\mathrm{D}$ in bone health [8]. While alone they may not significantly affect bone mineral density 
Table 3. Comparison of Changes in Bone Mineral Density From Baseline Values in Our Patients After 24 Months of Teriparatide, Calcium Citrate and Supraphysiologic Doses of Vitamin D; With Patients Treated With Teriparatide, Calcium and Physiologic Doses of Vitamin $D^{a}$

Patients treated with teriparatide and physiologic doses of vitamin D
Patient \#1 Patient \#2
Percent Change in BMD at

the Lumbar Spine over 24 months

Absolute increase in $\mathrm{g} / \mathrm{cm}^{2}$

over 24 months
$9.7 \%$

$48.2 \%$

0.189

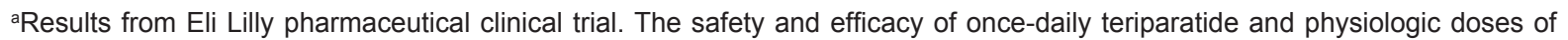
vitamin $\mathrm{D}$ with a median exposure of 19 months were examined in a double-blind, multicenter, placebo-controlled clinical trial of 1,637 postmenopausal women with osteoporosis [2].

or the risk of fractures, an adequate dose of both calcium and vitamin D complements the anti-fracture efficacy of the antiresorptive and anabolic agents used to treat osteoporosis $[3,12]$.

The Endocrine Society guidelines for vitamin D supplementation in patients at risk for vitamin $\mathrm{D}$ deficiency suggest $600 \mathrm{IU} /$ day for adults between the ages of 50 and 70, and $800 \mathrm{IU} /$ day for adults above the age of 70 [13]. For all adults with vitamin $\mathrm{D}$ deficiency, the guidelines suggest supplementation with $50,000 \mathrm{IU}$ of vitamin D once a week for 8 weeks or its equivalent of $6,000 \mathrm{IU} /$ day to achieve a minimum 25-hydroxyvitamin D serum level above $75 \mathrm{nmol} / \mathrm{L}$, followed by maintenance therapy of 1,500 to $2,000 \mathrm{IU} /$ day [13]. Despite such guidelines, there appears to be little information regarding how much vitamin D supplementation should be given to a postmenopausal female with osteoporosis requiring treatment with antiresorptive or anabolic agents.

Previous trials involving teriparatide therapy supplemented with vitamin $\mathrm{D}$ and calcium involve doses of vitamin D no higher than $1,200 \mathrm{IU} /$ day or with the aim of achieving a serum 25-hydroxyvitamin D level of $50 \mathrm{nmol} / \mathrm{L}$ [4]. Furthermore, previous studies involving the use of teriparatide in conjunction with vitamin $\mathrm{D}$ and calcium supplementation have suggested that vitamin D sufficiency may not be required to achieve an effective response to teriparatide [14]. In a study by Dawson-Hughes et al, patients received physiologic doses of vitamin D and calcium supplementation with teriparatide, and were compared to a placebo group who received similar doses of calcium and vitamin $\mathrm{D}$ without the intervention of teriparatide [14]. Their results suggested that responses to teriparatide did not differ significantly in women with baseline vitamin D insufficiency or sufficiency in regards to the endpoint of increased bone mineral density and reduction in vertebral and non-vertebral fracture risk [14]. However, in this study, baseline 25-hydroxyvitamin D levels were not significantly different between the vitamin D insufficient and sufficient groups, and the change in serum 25-hydroxyvitamin D concentrations from baseline values were not reported. Dawson-Hughes et al also observed an increased incidence of vitamin D insufficiency in all patient groups being treated with teriparatide after 12 months of teriparatide therapy, while this effect was not observed in the placebo group [14]. Perhaps there was no significant difference in bone mineral density or reduction in fractures observed between the vitamin D insufficient and sufficient groups because many patients became vitamin $\mathrm{D}$ insufficient after 12 months of teriparatide therapy. This may suggest the need for higher doses of vitamin D supplementation in patients being treated with teriparatide.

Vitamin D deficiency or insufficiency may lead to an increase in endogenous PTH levels, in order to maintain serum calcium levels within a normal range. This could potentially interfere with the action of teriparatide, a PTH analog. The actions of teriparatide on the bone seem to be effective due to its ability to quickly reach peak serum concentrations and decline to non-detectable levels within a few hours [7]. If endogenous PTH levels were to rise in the serum due to vitamin D deficiency or insufficiency, one cannot be certain that teriparatide can effectively achieve its full potential on bone remodeling. Raising 25-hydroxyvitamin D to a level that is well in the sufficient range may suppress endogenous PTH concentrations [15]. This could potentially minimize effects of endogenous PTH on bone remodeling, thus allowing teriparatide to achieve its full potential on bone remodeling in an osteoporotic patient.

Although the advantages of using supraphysiologic doses of vitamin D3 in conjunction with teriparatide are evident, one must be aware of the potential adverse effects of high dose vitamin D. Vitamin D toxicity has been known to cause hypercalcemia, hypercalciuria, as well as accelerated bone resorption. The lowest observed adverse effect level is 
the lowest serum concentration of 25-hydroxyvitamin D that produces signs or symptoms of vitamin D toxicity, including hypercalcemia or hypercalciuria. Many studies have shown that even with daily doses of vitamin D exceeding 40,000 IU, signs of vitamin D toxicity such as hypercalcemia only occurred in those individuals whose serum concentrations of 25-hydroxyvitamin D exceeded $750 \mathrm{nmol} / \mathrm{L}$ [15-18].

In trials testing the effect of high dose vitamin D supplementation on serum concentrations of 1, 25-dihydroxyvitamin D and calcium, it was found that even at daily doses of 10,000 IU of vitamin D for several weeks, there was no increase noted in the serum concentrations of calcium or 1, 25-dihydroxyvitamin $\mathrm{D}$, though a significant increase in serum 25-hydroxyvitamin D was produced [16, 19]. There were also no signs of toxicity associated with these high doses of vitamin D supplementation, including hypercalcemia or hypercalciuria $[16,19]$. In another study, daily doses of 50,000 IU of vitamin D for 8 weeks showed no evidence of vitamin D toxicity or changes in serum calcium or 1, 25-dihydroxyvitamin D levels, even with serum concentrations of 25-hydroxyvitamin D exceeding $600 \mathrm{nmol} / \mathrm{L}[19,20]$. This suggests that increased concentrations of 1, 25-dihydroxyvitamin D, the biologically active form of vitamin D, is what leads to vitamin D toxicity in which signs and symptoms of hypercalcemia and hypercalciuria are observed. Serum concentrations of 1,25-dihydroxyvitamin D are unlikely to change dramatically with significant increases in 25-hydroxyvitamin $\mathrm{D}$ due to tight regulation of renal conversion of 25-hydroxyvitamin D to 1, 25-dihydroxyvitamin D. Both parathyroid hormone and 1, 25-dihydroxyvitamin D itself, inhibit the conversion to active vitamin D [8].

In addition to this, most of the toxicity data regarding vitamin D is in relation to vitamin D2, not vitamin D3. Vitamin D2 has more of a propensity to cause toxic effects than vitamin D3 for several reasons. Vitamin D2 is a synthetic compound, while vitamin D3 is the naturally occurring form in humans. Therefore, there was greater impurity seen with vitamin D2 preparations [21]. In addition, because vitamin D2 is an artificial compound, the vitamin D binding protein has a weaker affinity for the metabolites of vitamin D2 in comparison to vitamin D3. This causes higher biologically available levels of free 25-hydroxyvitamin D2 and 1, 25-dihydroxyvitamin D2, leading to potential vitamin D toxicity at high daily doses [22, 23].

Taking into account the available data regarding toxicity of vitamin D, patients can be safely treated with supraphysiologic doses of vitamin D3 in conjunction with teriparatide, as long as serum concentrations of 25-hydroxyvitamin D, 1, 25-dihydroxyvitamin D, calcium, renal function, glomerular filtration rate, and 24-hour urine calcium are periodically measured during treatment. If nephrolithiasis in the setting of hypercalcemia and hypercalciuria is suspected, renal ultrasound can also be performed. Patients should also be counseled on the signs and symptoms seen with vitamin D toxicity, and if they exhibit signs of hypercalcemia or hypercalciuria, this should prompt an urgent appointment with a physician.

Not all patients receiving teriparatide may require supraphysiologic doses of vitamin D3. This treatment regimen may be best suited for patients who are vitamin D insufficient or deficient at the onset of therapy with teriparatide, as patients may exhibit worsening of vitamin D insufficiency or deficiency while receiving therapy with teriparatide. Furthermore, treatment with supraphysiologic doses of vitamin D3 in this subset of patients may suppress endogenous parathyroid hormone levels, allowing teriparatide to achieve its full potential on bone remodeling, without the possible interference of elevated endogenous levels of parathyroid hormone. After discontinuation of teriparatide, vitamin D supplementation should be decreased to physiologic doses.

\section{Conclusion}

No randomized, placebo controlled trials to date have shown the effects of supraphysiologic doses of vitamin D supplementation in conjunction with teriparatide therapy on bone mineral density and fracture prevention. Prior clinical studies of teriparatide given in conjunction with physiologic doses of calcium and vitamin D supplementation show that increases in BMD at the lumbar spine $[2,14]$. Although such increases in bone mineral density with the use of teriparatide are encouraging, further studies with teriparatide involving supraphysiologic doses of vitamin D should be considered. During these trials, serum 25-hydroxyvitamin D, 1, 25-dihydroxyvitamin $\mathrm{D}$, calcium, renal function, glomerular filtration rate, and 24-hour urine calcium should be monitored, as well as assessment of bone mineral density and fracture incidence. Clinicians should consider the possibility that raising 25-hydroxyvitamin D levels to well above borderline sufficient levels may have the beneficial effect of potentiating the actions of teriparatide on bone remodeling. This may potentially lead to greater increases in bone mineral density, and subsequently result in a further reduction in fracture risk.

\section{References}

1. Osteoporosis prevention, diagnosis, and therapy. NIH Consens Statement. 2000;17(1):1-45.

2. Forteo (teriparatide, Eli Lilly and Company, Indianapolis, IN). Full prescribing information, 2002

3. Hamdy RC, Chesnut CH, 3rd, Gass ML, Holick MF, Leib ES, Lewiecki ME, Maricic M, et al. Review of treatment modalities for postmenopausal osteoporosis. South Med J. 2005;98(10):1000-1014; quiz 1015-1007, 1048.

4. Han SL, Wan SL. Effect of teriparatide on bone mineral density and fracture in postmenopausal osteoporosis: 
meta-analysis of randomised controlled trials. Int J Clin Pract. 2012;66(2):199-209.

5. Ensrud KE, Thompson DE, Cauley JA, Nevitt MC, Kado DM, Hochberg MC, Santora AC, 2nd, et al. Prevalent vertebral deformities predict mortality and hospitalization in older women with low bone mass. Fracture Intervention Trial Research Group. J Am Geriatr Soc. 2000;48(3):241-249.

6. Hodsman AB, Bauer DC, Dempster DW, Dian L, Hanley DA, Harris ST, Kendler DL, et al. Parathyroid hormone and teriparatide for the treatment of osteoporosis: a review of the evidence and suggested guidelines for its use. Endocr Rev. 2005;26(5):688-703.

7. Deal C, Gideon J. Recombinant human PTH 1-34 (Forteo): an anabolic drug for osteoporosis. Cleve Clin $\mathrm{J}$ Med. 2003;70(7):585-586, 589-590, 592-584 passim.

8. Institute of Medicine. Dietary reference intakes for calcium and vitamin D. Washington, DC: The National Academies Press. 2011; 1015. http://books.nap.edu/openbook. php?record_id=13050. Accessed January 26, 2012.

9. Hollis BW. Circulating 25-hydroxyvitamin D levels indicative of vitamin D sufficiency: implications for establishing a new effective dietary intake recommendation for vitamin D. J Nutr. 2005;135(2):317-322.

10. Heaney RP, Dowell MS, Hale CA, Bendich A. Calcium absorption varies within the reference range for serum 25-hydroxyvitamin D. J Am Coll Nutr. 2003;22(2):142146.

11. Heaney RP. Functional indices of vitamin D status and ramifications of vitamin D deficiency. Am J Clin Nutr. 2004;80(6 Suppl):1706S-1709S.

12. Lips P, Graafmans WC, Ooms ME, Bezemer PD, Bouter LM. Vitamin D supplementation and fracture incidence in elderly persons. A randomized, placebo-controlled clinical trial. Ann Intern Med. 1996;124(4):400-406.

13. Holick MF, Binkley NC, Bischoff-Ferrari HA, Gordon CM, Hanley DA, Heaney RP, Murad MH, et al. Evalua- tion, treatment, and prevention of vitamin D deficiency: an Endocrine Society clinical practice guideline. J Clin Endocrinol Metab. 2011;96(7):1911-1930.

14. Dawson-Hughes B, Chen P, Krege JH. Response to teriparatide in patients with baseline 25-hydroxyvitamin D insufficiency or sufficiency. J Clin Endocrinol Metab. 2007;92(12):4630-4636.

15. Vieth R. Vitamin D supplementation, 25-hydroxyvitamin D concentrations, and safety. Am J Clin Nutr. 1999;69(5):842-856.

16. Heaney RP, Davies KM, Chen TC, Holick MF, BargerLux MJ. Human serum 25-hydroxycholecalciferol response to extended oral dosing with cholecalciferol. Am J Clin Nutr. 2003;77(1):204-210.

17. Koutkia P, Chen TC, Holick MF. Vitamin D intoxication associated with an over-the-counter supplement. N Engl J Med. 2001;345(1):66-67.

18. Breslau NA, Zerwekh JE. Pharmacology of vitamin D preparations. In: Feldman D, Glorieux FH, Pike JW, eds. Vitamin D. New York: Academic Press, 1997: 607-18

19. Barger-Lux MJ, Heaney RP, Dowell S, Chen TC, Holick MF. Vitamin D and its major metabolites: serum levels after graded oral dosing in healthy men. Osteoporos Int. 1998;8(3):222-230.

20. Glade MJ. A 21st century evaluation of the safety of oral vitamin D. Nutrition. 2012;28(4):344-356.

21. Bicknell F, Prescott F. Vitamin D. The antirachitic or calcifying vitamin. In: Bicknell F, Prescott F, eds. Vitamins in medicine. London: Whitefriars Press, 1946: 630-707.

22. Jones G, Byrnes B, Palma F, Segev D, Mazur Y. Displacement potency of vitamin D2 analogs in competitive protein-binding assays for 25-hydroxyvitamin D3, 24,25-dihydroxyvitamin D3, and 1,25-dihydroxyvitamin D3. J Clin Endocrinol Metab. 1980;50(4):773-775.

23. Vieth R, Chan PC, MacFarlane GD. Efficacy and safety of vitamin D3 intake exceeding the lowest observed adverse effect level. Am J Clin Nutr. 2001;73(2):288-294. 